University of New Hampshire

University of New Hampshire Scholars' Repository

Space Science Center

Institute for the Study of Earth, Oceans, and

Space (EOS)

8-20-2009

\title{
GRAPE: a balloon-borne gamma-ray polarimeter
}

\author{
Mark L. McConnell \\ mark.mcconnell@unh.edu \\ Chris Bancroft \\ University of New Hampshire \\ Peter F. Bloser \\ University of New Hampshire, Peter.Bloser@unh.edu \\ Taylor Connor \\ University of New Hampshire - Manchester \\ Jason S. Legere \\ University of New Hampshire, jslegere@unh.edu
}

See next page for additional authors

Follow this and additional works at: https://scholars.unh.edu/ssc

Part of the Astrophysics and Astronomy Commons

\section{Recommended Citation}

Mark L. McConnell ; Christopher Bancroft ; Peter F. Bloser ; Taylor Connor ; Jason Legere and James M. Ryan "GRAPE: a balloon-borne gamma-ray polarimeter", Proc. SPIE 7435, UV, X-Ray, and Gamma-Ray Space Instrumentation for Astronomy XVI, 74350J (August 20, 2009); doi:10.1117/12.826407; http://dx.doi.org/10.1117/12.826407

This Conference Proceeding is brought to you for free and open access by the Institute for the Study of Earth, Oceans, and Space (EOS) at University of New Hampshire Scholars' Repository. It has been accepted for inclusion in Space Science Center by an authorized administrator of University of New Hampshire Scholars' Repository. For more information, please contact Scholarly.Communication@unh.edu. 
Authors

Mark L. McConnell, Chris Bancroft, Peter F. Bloser, Taylor Connor, Jason S. Legere, and James M. Ryan 


\title{
GRAPE : A balloon-borne gamma-ray polarimeter
}

\author{
Mark L. McConnell' ${ }^{1}$ Christopher Bancroft, Peter F. Bloser, \\ Taylor Connor, Jason Legere, and James M. Ryan \\ Space Science Center, University of New Hampshire, Durham, NH 03824
}

\begin{abstract}
The Gamma-RAy Polarimeter Experiment (GRAPE) is a concept for an astronomical hard X-ray Compton polarimeter operating in the $50-500 \mathrm{keV}$ energy band. The instrument has been optimized for wide-field polarization measurements of transient outbursts from energetic astrophysical objects such as gamma-ray bursts and solar flares. The GRAPE instrument is composed of identical modules, each of which consists of an array of scintillator elements read out by a multi-anode photomultiplier tube (MAPMT). Incident photons Compton scatter in plastic scintillator elements and are subsequently absorbed in inorganic scintillator elements; a net polarization signal is revealed by a characteristic asymmetry in the azimuthal scattering angles. We have constructed a prototype GRAPE module that has been calibrated at a polarized hard X-ray beam and flown on an engineering balloon test flight. A full-scale scientific balloon payload, consisting of up to 36 modules, is currently under development. The first flight, a one-day flight scheduled for 2011, will verify the expected scientific performance with a pointed observation of the Crab Nebula. We will then propose long-duration balloon flights to observe gamma-ray bursts and solar flares.
\end{abstract}

Keywords: X-ray, gamma-ray, scintillator, astronomy, Compton, polarimetry, balloon

\section{INTRODUCTION}

The Gamma-RAy Polarimeter Experiment (GRAPE) is a scintillator-based Compton polarimeter designed to observe polarized astrophysical phenomena in the hard X-ray energy band $(50-500 \mathrm{keV})$. Although intended primarily for observations of bright, transient events such as gamma-ray bursts (GRBs) and solar flares, GRAPE may also be operated in a collimated, pointed mode. The basic instrument concept has been validated in laboratory experiments and during a calibration campaign at a polarized X-ray beam, and the hardware has been flight-tested on an engineering balloon flight. We are currently developing a full-scale scientific payload for a one-day balloon flight, and plan to expand this instrument for long-duration balloon flights in the coming years while pursuing opportunities for an orbital platform.

The primary science goal of GRAPE is to measure polarization from energetic transient sources, such as GRBs and solar flares, in order to study to physics of particle acceleration. The prompt gamma ray emission in GRBs is believed to result from particles accelerated by shocks in a highly relativistic jet aimed at the observer. Polarization measurements will help to distinguish between two basic classes of models [1]. In "physical" models, a globally ordered magnetic field produces high levels of polarization via synchrotron radiation, with only a weak dependence on viewing geometry. In "geometric" models a randomly tangled magnetic field can yield high polarization only if the event is viewed very close to the edge of the jet, due to relativistic beaming. These models can be distinguished by observing a large sample of GRBs and measuring the fraction displaying significant polarization [1]. Solar flares represent a process of explosive energy release in the magnetized plasma of the solar corona, with electrons being accelerated to hundreds of $\mathrm{MeV}$. The details of how the Sun releases this energy and efficiently transfers it into accelerated par-

${ }^{1}$ Correspondence: mark.mcconnell@unh.edu

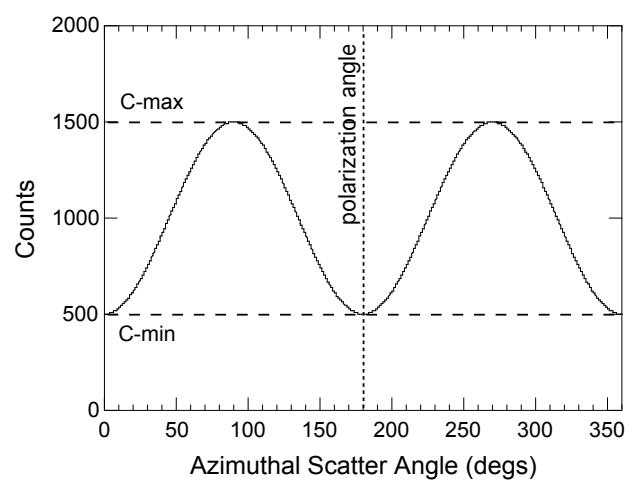

Figure 1: The modulation pattern produced by Compton scattering of polarized radiation. The minimum of the modulation pattern defines the plane of polarization of the incident flux.

UV, X-Ray, and Gamma-Ray Space Instrumentation for Astronomy XVI, edited by Oswald H. Siegmund, Proc. of SPIE Vol. 7435, 74350J - (c) 2009 SPIE CCC code: 0277-786X/09/\$18 - doi: 10.1117/12.826407 
ticles are unknown. Polarization measurements of the hard X-ray emission from flares, due to bremsstrahlung from these energetic electrons, are expected to be useful in determining the beaming, or anisotropy, of these electrons [2, 3], which will provide clues about electron acceleration and transport mechanisms.

\section{COMPTON POLARIMETRY}

The physical process used to measure polarization in the 50-500 keV energy range is Compton scattering. Compton scattered photons tend to be scattered at right angles with respect to the incident electric field. In the case of an unpolarized beam of incident photons, there will be no preferred azimuthal scattering angle $(\eta)$; the distribution of scattered photon angles with respect to $\eta$ will therefore be uniform. However, in the polarized case, the incident photons will exhibit an asymmetric distribution in $\eta$ (Figure 1). The magnitude of the asymmetry is largest at low energies (below $100 \mathrm{keV}$ ) and is most pronounced for Compton scatter angles $(\theta)$ near $90^{\circ}$.

In general, a Compton scatter polarimeter consists of two detectors to determine the energies of both the scattered photon and the scattered electron. The scattering detector provides the medium for the Compton interaction to take place. This also requires a low- $\mathrm{Z}$ material to minimize photoelectric interactions. The second detector, the calorimeter, absorbs the full energy of the scattered photon. The relative placement of the two detectors defines the scattering geometry and permits a measurement of the azimuthal scatter angle $(\eta)$. The distribution of azimuthal scatter angles follows a $\cos 2 \eta$ distribution (Figure 1),

$$
C(\eta)=A \cos \left[2\left(\eta-\varphi+\frac{\pi}{2}\right)\right]+B
$$

where $\phi$ is the polarization angle of the incident photons; A and B are constants. The quality of the polarization signal is quantified by the polarization modulation factor [4]. For a given energy and incidence angle, this can be expressed as,

$$
\mu_{p}=\frac{C_{p, \text { max }}-C_{p, \text { min }}}{C_{p, \text { max }}+C_{p, \text { min }}}=\frac{A_{p}}{B_{p}}
$$

where $C_{p, \max }$ and $C_{p, \min }$ refer to the maximum and minimum number of counts registered in the polarimeter, respectively, with respect to $\eta ; A_{p}$ and $B_{p}$ refer to the corresponding parameters in equation (1). In this case the 'p' subscript denotes that this refers to the measurement of a beam with unknown polarization. In order to determine the polarization of the measured beam, we need to know (e.g., from simulations) how the polarimeter would respond to a similar beam, but with $100 \%$ polarization $\left(\mu_{100}\right)$. We can then use this result, in conjunction with the observed modulation factor $\left(\mu_{\mathrm{P}}\right)$, to determine the level of polarization (П) in a measured beam [4],

$$
\Pi=\mu_{p} / \mu_{100}
$$

The minimum detectable polarization (MDP) is expressed as [5],

$$
\operatorname{MDP}(\%)=\frac{n_{\sigma}}{\mu_{100} R_{s r c}} \sqrt{\frac{R_{s r c}+R_{b g d}}{T}}
$$

where $n_{\sigma}$ is the significance level (number of sigma), $R_{\text {src }}$ is the source counting rate, $R_{b g d}$ is the background counting rate (determined independently) and $\mathrm{T}$ is the observation time. From this expression, we can then define a relative figureof-merit (FoM) as,

$$
F o M=\mu \sqrt{A_{e f f}}
$$

where $A_{\text {eff }}$ is the effective area. This can be useful in comparing different instruments or instrument configurations. 


\section{THE GRAPE DESIGN}

The GRAPE design has evolved through multiple iterations. In addition to an early prototype polarimeter, we have tested several different science model designs (SM1, SM2, and SM3) and one engineering model (EM1). We distinguish between a science model and an engineering model based on the readout electronics. The science models relied on commercial laboratory electronics (NIM, CAMAC, VME) and software (Kmax or LabView). The engineering model employs customdesigned, compact, low-power electronics, custom software and a stand-alone custom-configured computer that provides autonomous control, coincidence determination and data acquisition. All of these designs consist of an array of scintillators placed on the front end of a single photo-multiplier tube (PMT). Two types of scintillator are used. Low-Z plastic scintillator elements (scattering elements) are used as the medium for Compton scattering the incident photons. High- $Z$ inorganic scintillator crystals (calorimeter elements) are used as a medium for maximizing the absorption probability of the scattered photon. In each case, the scintillator elements are arranged so that the azimuthal scatter angle of photons that scatter between a scattering element and a calorimeter element can be determined. Figure 2 depicts a typical configuration with both inner plastic elements and outer calorimeter elements. A valid event involves the interaction of an incident photon in a plastic element followed by the absorption of the scattered photon in a calorimeter element. The locations of the two hit elements define the azimuthal scatter angle (with respect to some fixed reference). The details of the various models are summarized in Table 1. Figure 3 shows each science model in the lab.

For the plastic scattering elements, we have used either BC-404 (Saint Gobain Cystals) or its equivalent, EJ-204 (Eljen Technology). For the calorimeter elements, we have baselined the use of CsI(Na) scintillator. The CsI(Na) was chosen for its good spectral match to traditional bi-alkali photocathodes, its good light output and its relatively low cost. Another reason for selecting CsI $(\mathrm{Na})$ is its decay time. In order to deal effectively with optical crosstalk between different type elements on a single phototube, we have developed pulse-shape discrimination circuitry for distinguishing events in adjacent scattering and calorimeter elements. This technique relies on there being a significant difference in decay times between the two scintillator types. CsI(Na) has a decay time $(600$ $\mathrm{nsec})$ that is significantly different from that of plastic scintillator $(1.8 \mathrm{nsec})$ and therefore meets this requirement. Other materials that might be more attractive in terms of energy resolution (such as $\mathrm{LaBr}_{3}$ ) have much shorter decay constants $(\sim 20 \mathrm{nsec})$ that make the PSD approach difficult to implement. CsI $(\mathrm{Na})$ provides adequate energy resolution for effective scientific studies.

The initial SM1 design utilized a single 5-inch diameter position-sensitive PMT (PSPMT - Hamamatsu R3292) to read out a large array of plastic scattering elements and an independent multi-anode PMT (MAPMT) to read out an array of four 1-cm CsI(Na) scintillators [6-13]. All subsequent GRAPE science models (Figure 3) and the engineering model
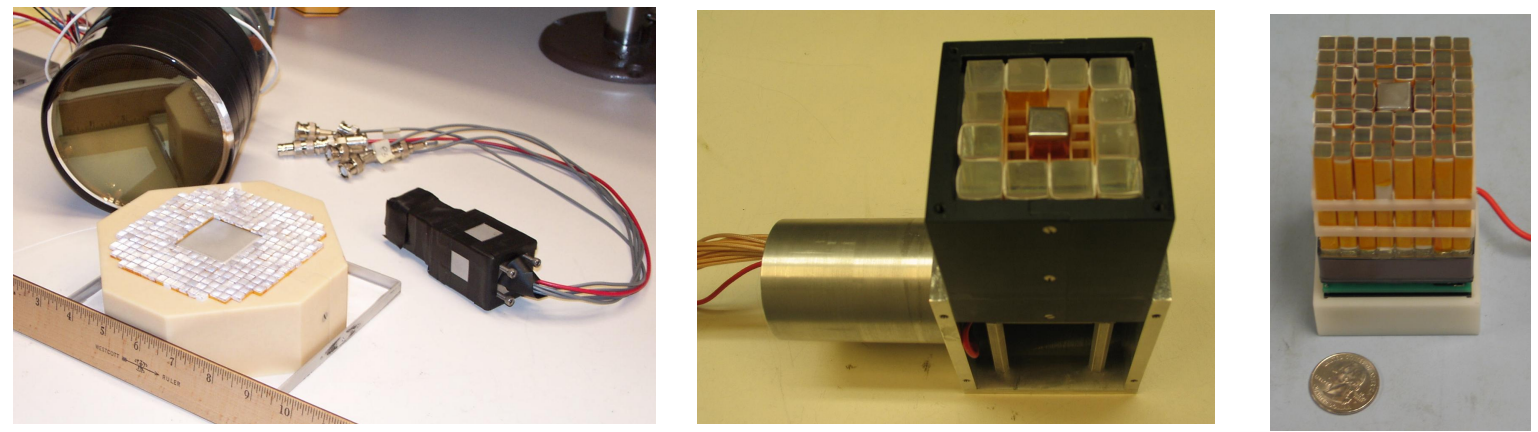

Figure 3: The components of SM1 (left), SM2 (middle) and SM3 (right). SM1 used a 5-inch PSPMT. Both SM2 and SM3 used a 2-inch MAPMT, but with different scintillator arrangements. 
TABLE 1: History of GRAPE Development

\begin{tabular}{|l|l|l|}
\hline Design & \multicolumn{1}{|c|}{ PMT } & \multicolumn{1}{c|}{ Scintillator } \\
\hline SM1 & $\begin{array}{l}\text { 5" position-sensitive PMT } \\
\text { (Hamamatsu R3292) }\end{array}$ & $\begin{array}{l}\text { 5-mm square plastic [250] } \\
\text { 1-cm square CsI(Na) [4] }\end{array}$ \\
\hline SM2 & $\begin{array}{l}\text { 5-cm flat-panel MAPMT } \\
\text { (Hamamatsu H8500) }\end{array}$ & $\begin{array}{l}\text { 1-cm square plastic [12] } \\
\text { 1-cm square CsI(Na) [1] }\end{array}$ \\
\hline SM3 & $\begin{array}{l}\text { 5-cm flat-panel MAPMT } \\
\text { (Hamamatsu H8500) }\end{array}$ & $\begin{array}{l}\text { 5-mm square plastic [60] } \\
\text { 1-cm square CsI(Na) [1] }\end{array}$ \\
\hline EM1 & $\begin{array}{l}\text { 5-cm flat-panel MAPMT } \\
\text { (Hamamatsu H8500) }\end{array}$ & $\begin{array}{l}\text { 5-mm square plastic [60] } \\
\text { 1-cm square CsI(Na) [1] }\end{array}$ \\
\hline
\end{tabular}

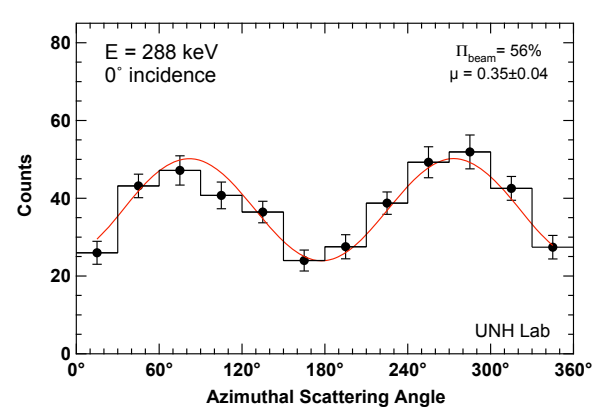

Figure 4: Laboratory result from SM3 (at $288 \mathrm{keV}$ ). Smooth curve represent a fit to the data. The polarization angle corresponds to the minimum of the modulation pattern.

have all relied on a single flat-panel MAPMT (Hamamatsu H8500) for simultaneous readout of both plastic and calorimeter elements [14-20]. The H8500 provides an $8 \times 8$ array of independent anodes, with $5 \mathrm{~mm}$ anodes arranged on a pitch of $6 \mathrm{~mm}$. Scintillator elements (either plastic or $\mathrm{CsI}(\mathrm{Na})$ ) are co-aligned for readout by a single anode. The MAPMT itself measures $52 \times 52 \mathrm{~mm}^{2}$ in area and $28 \mathrm{~mm}$ in depth, far more compact than the PSPMT used in SM1. The square design of the MAPMT also allows for the close packing of multiple modules with minimal dead space - a significant advantage for many applications.

For each design, measurements were conducted to evaluate the response of the detector to polarized radiation. In the laboratory, a source of polarized photons was generated by Compton scattering photons from a radioactive source. The level of polarization of such a scattered photon beam is dependent on both the initial photon energy and the photon scatter angle [21]. The $90^{\circ}$ scattering of photons from a ${ }^{137} \mathrm{Cs}$ source, for example, generates $288 \mathrm{keV}$ photons with a polarization fraction of $\sim 55 \%$. The use of plastic scintillator as a scattering block to generate the polarized beam permits the electronic tagging of the scattered (polarized) photons. A triple coincidence between the polarizer and the two sets of polarimeter detectors (scattering and calorimeter elements) identifies interactions of polarized photons and provides an efficient means for recording data. We have performed laboratory calibrations for all four designs at various energies, incidence angles and polarization angles (e.g., Figure 4). The purpose of these calibrations was not only to demonstrate the efficacy of the design, but also to validate the simulation tools. Our simulations rely on the use of MGEANT [22], which uses the GLEPS ${ }^{2}$ package to model the effects of polarization in Compton scattering.

\section{ENGINEERING MODEL}

An effort to design, fabricate and test an MAPMT-based polarimeter module and associated electronics for balloon program applications resulted in a fully functioning 64channel module assembly, which we refer to as EM1 (Figure 5). The EM1 assembly (including the associated electronics) can easily be modified to accommodate any configuration of scintillator elements within the $8 \times 8$ array, along with signals from external detectors such as active shield panels in anti-coincidence. The module electronics handles fast and slow signal processing, pulse shape discrimination to reduce crosstalk among neighboring scintillator elements of different species, internal and external fast coincidence, digital conversion of pulse heights, sparse readout of the event data, collection of local house-keeping data (rates, temperatures, etc.) and handling of all communications (data and commands) with the instrument computer. Total power is $1.8 \mathrm{~W}$ per 64 channel module. The design was intentionally developed without the use of custom ASICs to assure flexibility with evolving balloon instrument configurations while minimizing the cost of future modifications.

2 http://public.lanl.gov/mkippen/actsim/glecs/

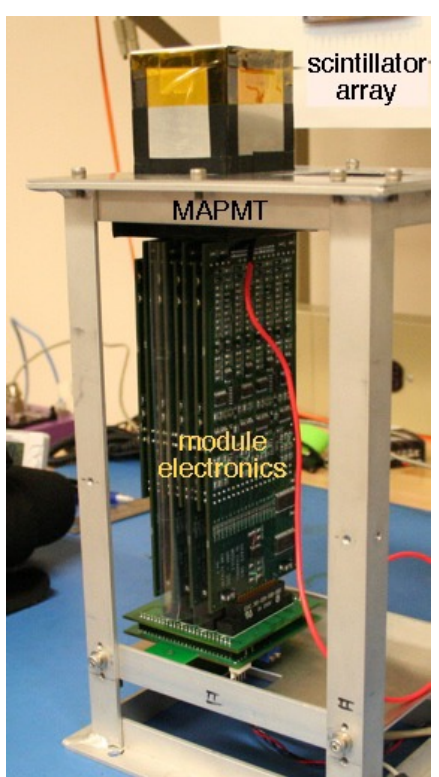

Figure 5: The GRAPE engineering model (EM1). 


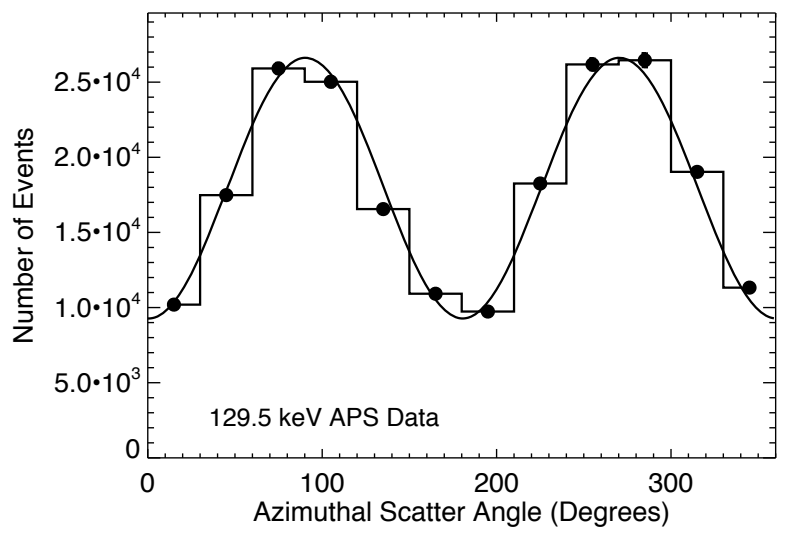

Figure 6: Polarization signature recorded with EM1 at Argonne National Laboratory using polarized photon beam at $129 \mathrm{keV}$. Error bars are too small to be seen.

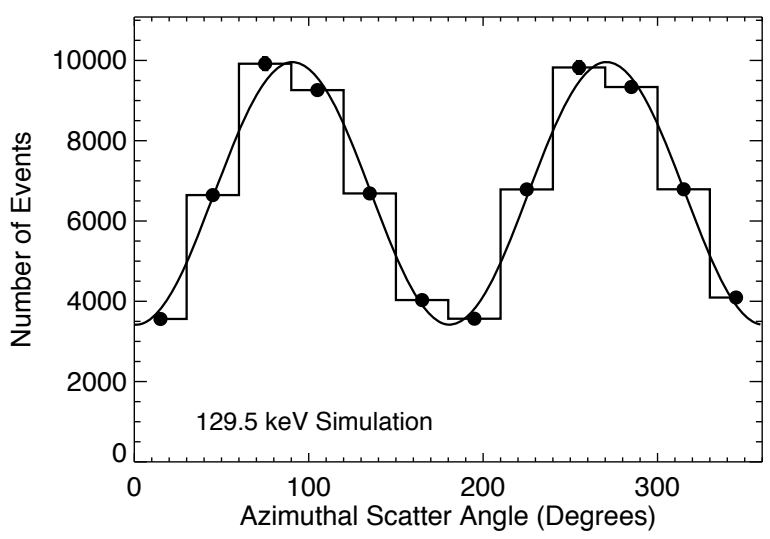

Figure 7: Polarization signature recorded with EM1 at Argonne National Laboratory using polarized photon beam at $129 \mathrm{keV}$. Error bars are too small to be seen.

\subsection{Beam Calibration}

The GRAPE EM1 was calibrated in December 2006 using a hard X-ray beam at the Advanced Photon Source (APS) at Argonne National Laboratory in order to measure the modulation factor for nearly $100 \backslash \%$ polarized radiation and to validate our Monte Carlo simulations. The instrument was exposed to two energies, $69.5 \mathrm{keV}$ and $129.5 \mathrm{keV}$, with a polarization fraction calculated to be $97 \pm 2 \%$. As the beam was less than $1 \mathrm{~mm}$ in diameter it was necessary to expose one plastic element at a time using an $\mathrm{X}-\mathrm{Y}$ translation table. Alternate elements were exposed in a checkerboard pattern due to time constraints. After completing a scan, EM1 was rotated $90^{\circ}$ and the scan was repeated. In this way we were able to create an "unpolarized" exposure by combining data from both orientations relative to the polarization vector; these unpolarized data were used for the removal of geometric effects. The data for each plastic exposure were corrected for exposure time and beam flux and combined into azimuthal scatter angle histograms for the entire instrument.

The fitted azimuthal modulation histogram for $129.5 \mathrm{keV}$ is shown in Figure 6. The fitted modulation factor, assuming $97 \%$ polarized radiation, is $\mu_{97}=0.48 \pm 0.03$. The simulated modulation histogram is shown in Figure 7. For the simulation we find $\mu_{97}=0.49 \pm 0.02$, in excellent agreement with the data. These results show that GRAPE is a sensitive polarimeter, and that our Monte Carlo tools accurately reproduce its performance.

\subsection{Engineering Model Balloon Flight}

The EM1 instrument was fitted with passive
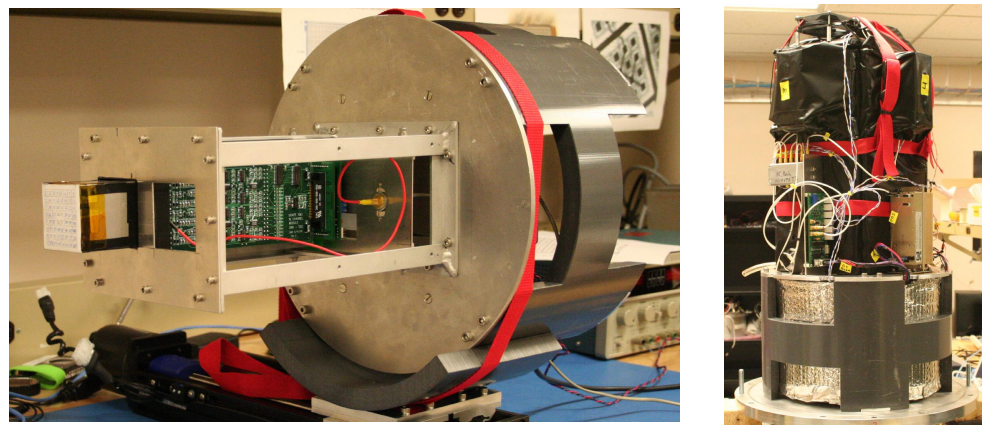

Figure 8: Two views of the GRAPE EM1 balloon package. The entire package was enclosed within a pressure vessel.

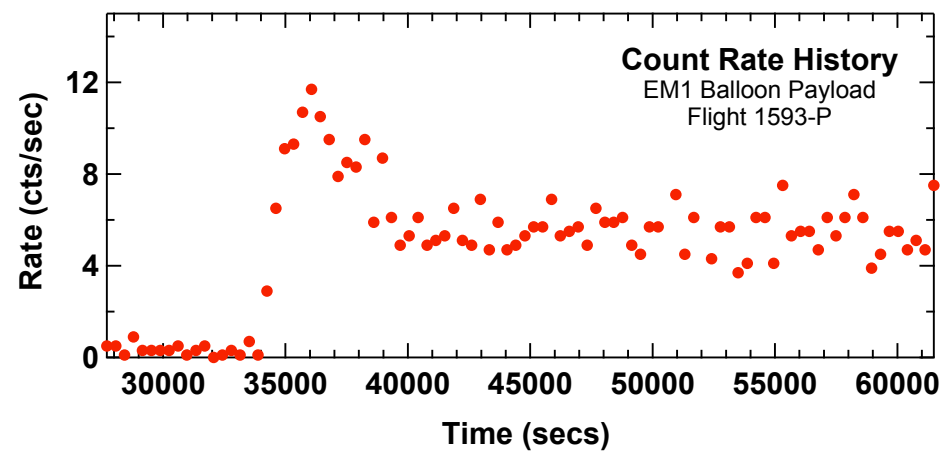

Figure 9: Rate meter history of the EM1 module during ascent through the Pfotzer maximum and at float altitude. This includes all events from 50-300 $\mathrm{keV}$ involving from 1-3 plastic elements (both vetoed and non-vetoed) along with events from an overflow channel. 
and active plastic shielding, sealed within a pressure vessel, and flown on an engineering balloon flight (Figure 8). The single GRAPE module did not provide sufficient sensitivity for any meaningful science (except perhaps in the case of an extremely large GRB or solar flare). The package was successfully flown on June 21, 2007 (Flight 1593-P) from Palestine, TX. It remained at float $(\sim 130,000 \mathrm{ft}$.) for 5.5 hours before termination. The payload, along with the on-board data, was recovered the following day. The count rate history is shown in Figure 9. These data indicated a stable 50-300 keV background rate (for valid events - those that trigger one plastic and one calorimeter element) of $\sim 1.1 \mathrm{cts} \mathrm{s}^{-1}$ per module and also demonstrated the viability of the GRAPE engineering design. The background spectrum was roughly consistent with a simple Monte Carlo simulation of the expected counts due to atmospheric and cosmic gamma rays. More detailed background simulations are in progress.

\section{BALLOON PAYLOAD}

We are currently preparing for the first balloon flight of a science-capable GRAPE payload. The first flight of the GRAPE payload will take place in the fall of 2011, from Ft. Sumner. This continental US (CONUS) turn-around flight will demonstrate the integrity of the payload, the polarimeter array and the associated electronics. With a configuration that provides $\sim 10^{\circ}$ collimation of a $4 \times 4$ module array, the primary science target will be the Crab. We will utilize the Crab polarization not only to achieve an important science goal, but also to validate the scientific capability of GRAPE. Secondary targets for this first flight include Cyg X-1 and, should there be significant solar activity at the time, the Sun.

If all goes well, subsequent flights (in Dec of 2012 and 2013) will take place from McMurdo station in Anatarctica as Long Duration Balloon (LDB) missions, each lasting 20-30 days. The payload for these flights will be configured as an un-collimated array $\left(\sim 120^{\circ} \mathrm{FoV}\right)$ to maximize the probability of observing GRBs. The detector array will be expanded each year so that we will have a total of 36 GRAPE modules for the final flight in 2013. Because this time frame corresponds to the solar maximum, we will operate the payload to provide moderate exposure to the Sun, with the array pointed towards a zenith angle of $\sim 30^{\circ}$ and with the pointing azimuth following the solar azimuth. Despite the Sun's low altitude (ranging from $10^{\circ}$ to $35^{\circ}$ at the latitude of McMurdo), the large FoV of the GRAPE array will provide continuous exposure to the Sun.

\subsection{Detector Configuration}

Simulations have been used to optimize the module design. Based on these studies, our payload will incorporate an arrangement of scintillator elements, as shown in Figure 10. Compared to earlier versions of GRAPE, this design increases the number of calorimeter elements and arranges them around the outside of the detector array. This geometry results in a significantly improved polarization sensitivity.

\subsection{Instrument Configuration}

The layout of the detector assembly follows that depicted in Figure 11 , with an array of up to 36 independent polarimeter modules. Each module assembly (Figure 11) has 36 independent plastic elements surrounded by $28 \mathrm{CsI}(\mathrm{Na})$ elements $(5 \times 5 \times 50 \mathrm{~mm} 3$ in size $)$ read out by a single Hamamatsu H8500 MAPMT. Each module has four (4) 16channel analog electronics boards to process the MAPMT anode signals. Each channel has a fast discriminator to generate triggers for the hit scintillator elements and a slow shaper with peak-detect-and-hold circuitry that forms part of the pulse height measurement. An interface board and a logic board with FPGA fast coincidence circuitry to recognize coincident and anti-coincident events, validate each event, multiplex and digitize the pulse heights and assemble event messages for further processing, complete the module electronics. Module housings are designed so that assembly, test, calibration and debug activities can be conducted on individual modules outside of the detector array. There is no provision for handling those events that scatter from a plas-

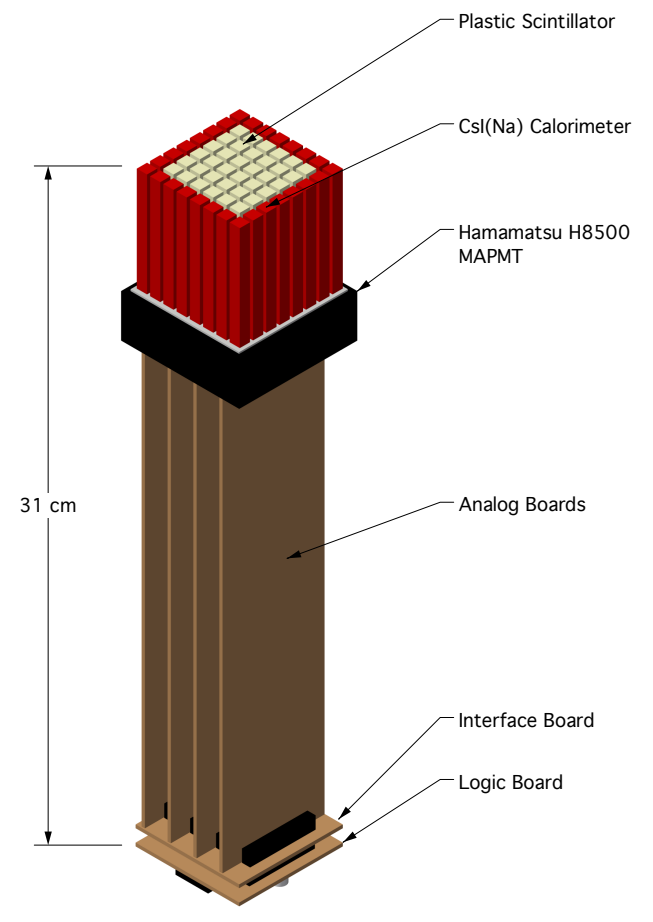

Figure 10: A GRAPE polarimeter module, showing the individual scintillator elements, the MAPMT, the layout of the four analog boards and their interface to the module logic board. 
tic element in one module into a calorimeter element in an adjacent module, since simulations show that there is a relatively small number of such events (primarily due to the stopping power of CsI that surrounds the central plastic elements). Although each polarimeter module provides a measure of the source energy spectrum, one of the polarimeter modules in the array may be substituted with a dedicated spectrometer module to augment these spectral data. This would consist of a single $50 \times 50$ x $50 \mathrm{~mm} 3 \mathrm{NaI}(\mathrm{Tl})$ detector read out by a $\mathrm{H} 8500$ MAPMT, providing spectral information from 15 $\mathrm{keV}$ to $1 \mathrm{MeV}$. The mechanical and electrical interfaces would be the same as for the polarimeter modules, thus simplifying the design.

The anticoincidence detector is composed of 6 rectangular panels mounted to an aluminum frame to surround the polarimeter array on its four sides, top and bottom. Each panel has a 5-mm thick sheet

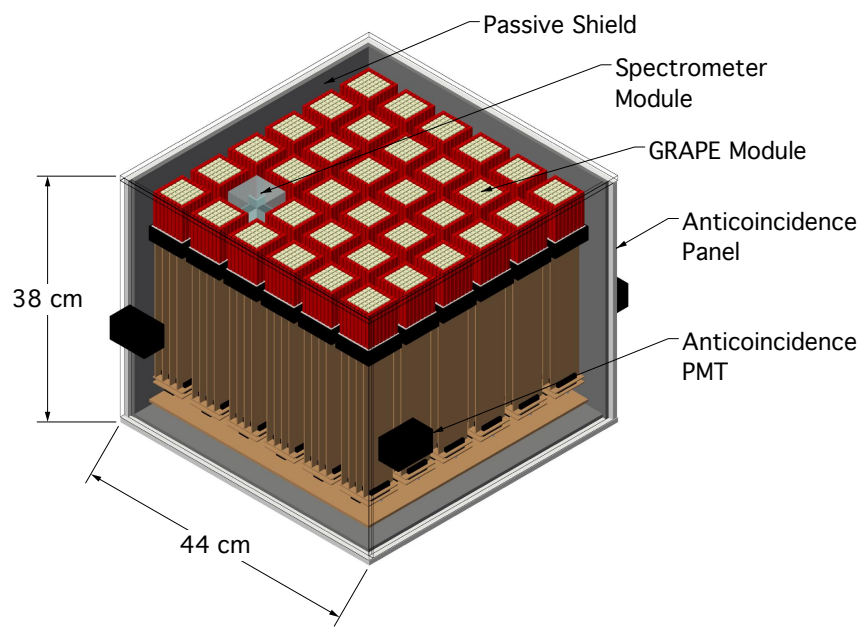

Figure 11: The fully-populated GRAPE detector assembly, shown with one polarimeter module replaced by a spectroscopy module. For the first two balloon flights, this array may be only partially-populated. of plastic scintillator contained in a rigid, light tight (honeycomb) aluminum housing. The design uses a wavelength shifting (WLS) bar and a single PMT to read out the signal from each scintillator panel. This approach was developed in laboratory studies at UNH for FiberGLAST [23] and was used for the EM1 balloon payload. It features easily handled modular panels and generates strong signals with relatively thin scintillator sheets.

A multi-layer passive shield $(\mathrm{Pb}, \mathrm{Sn}$ and $\mathrm{Cu})$ surrounds the polarimeter detector array on five sides to provide passive shielding. These layers attach to the inner walls of the side and bottom anticoincidence panels, with the copper facing the detector array. The passive shield serves to absorb atmospheric hard X-ray radiation from below and to the sides of the payload, with the inner, lower-Z layers blocking any fluorescent X-ray emission from the outer, higher-Z layers. For point source observations (in the CONUS flight), passive multi-layer absorbers $(\mathrm{Pb}, \mathrm{Sn}, \mathrm{Cu}$ ) will also be used to collimate the response of the detector array to within an acceptance angle of $\sim 10^{\circ}$. The collimators will surround each of the detector modules and extend $\sim 30 \mathrm{~cm}$ in front of the sensitive detector volume. The collimators will not be employed for the LDB flights, where wide FoV observations are required.

\subsection{Payload Configuration}

The balloon gondola (with a fully populated detector array) is illustrated in Figure 12. The instrument components are mounted inside a pressure vessel. An axial rotation mechanism, designed to mitigate the effects of instrumental systematics by rotating the instrument back and forth $\left( \pm 90^{\circ}\right)$ about the pointing direction, is also mounted inside the pressure vessel. An external frame (fabricated from extruded aluminum stock) supports and protects the instrument pressure vessel and provides the mounting structure for the remaining UNH and CSBF payload components. Mass, power and telemetry estimates are given in Table 2 for each of the three flight configurations. The pressure vessel is a three-piece aluminum assembly consisting of a cylindrical sidewall and upper and lower domes. It will contain one atmosphere (15 psia) of gaseous nitrogen (GN2). Instrument integration, test and debug activities can be conducted with the pressure vessel's top

TABLE 2: Estimated Payload Parameters

\begin{tabular}{|c|c|c|c|c|c|}
\hline Flight & Date & Site & $\begin{array}{c}\text { Total Mass } \\
\text { (w/o ballast) }\end{array}$ & Power & Avg Data Rate \\
\hline CONUS & Fall, 2011 & Ft. Sumner & $800 \mathrm{~kg}$ & $165 \mathrm{~W}+25 \mathrm{~W}$ heater & $9.0 \mathrm{kbs}$ \\
\hline LDB1 & Dec, 2012 & McMurdo & $1070 \mathrm{~kg}$ & $204 \mathrm{~W}+25 \mathrm{~W}$ heater & $14.0 \mathrm{kbs}$ \\
\hline LDB2 & Dec, 2013 & McMurdo & $1080 \mathrm{~kg}$ & $243 \mathrm{~W}+25 \mathrm{~W}$ heater & $19.0 \mathrm{kbs}$ \\
\hline
\end{tabular}


and bottom dome elements removed to facilitate access. The cylindrical sidewall is the structural element. All instrument hardware is supported from a base plate mounted to the sidewall. The detector modules, five anticoincidence panels and the motherboard mount to the top of this base plate; the bottom anticoincidence panel, the instrument computer and the instrument power supply and distribution module mount to the bottom side of this base plate. The sidewall has ports for electrical and gas feed-throughs and brackets for secure mounting to the frame. The pressure vessel's top and bottom dome elements are formed with $2-3 \mathrm{~mm}$ thick aluminum. The temperature within the pressure vessel will be maintained in the $-10^{\circ}$ to $+30^{\circ} \mathrm{C}$ range using fans and heaters.

The requirements of the attitude control system (ACS) are driven by the CONUS ballon flight, where collimated observations of point sources will be conducted. The CONUS requirement will be to maintain the source target within the $\sim 10^{\circ} \mathrm{FoV}$. Control precision of $\pm 3^{\circ}$ is adequate with pointing knowledge uncertainty of $\pm 0.5^{\circ}$. A reaction wheel and momentum transfer unit and an elevation drive mechanism will be employed. A commercial differential GPS unit will be used to provide the gondola's 3dimensional orientation at frequent intervals. A magnetometer-based digital compass and GPS sensor and

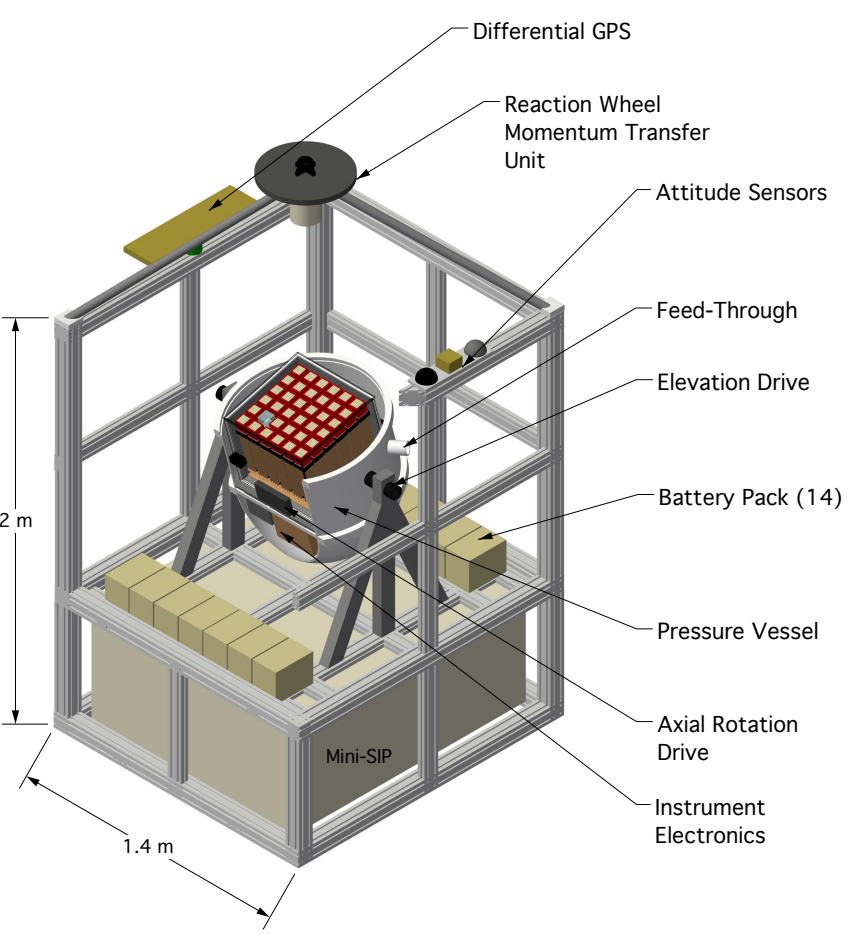

Figure 12: The GRAPE balloon gondola. The detector array and associated electronics fit within an aluminum pressure vessel. 3-axis Sun sensor will act as a backup. The elevation angle will be measured with encoders and recorded along with the time-tagged azimuth information.

An effective way to deal with the problem of instrumental systematics is to induce a rotation of the polarimeter about the pointing axis. Rather than having a continuous $360^{\circ}$ rotation, the payload will employ a simpler (and, for our purposes, equivalent) design that rotates the detector back and forth through a range of $180^{\circ}\left( \pm 90^{\circ}\right)$. A drive for axial rotation of the instrument will be implemented within the pressure vessel to provide a rotation rate of $\sim 180^{\circ}$ per minute. An encoder in the axial drive system will measure and record the time-tagged orientation as part of the housekeeping data; modest knowledge of this orientation $\left( \pm 1^{\circ}\right)$ is adequate.

The CONUS flight will utilize CSBF L- or S-band transmitters for line-of-sight (LOS) telemetry of the science data. These can handle up to $700 \mathrm{kbits} / \mathrm{s}$, sufficient for handling the expected rate of science data (Table 2). LDB flights will additionally employ the provided TDRSS and IRIDIUM telemetry systems for state of health monitoring, instrument commanding and relay of buffered GRB (and solar flare) science data during periods when LOS telemetry is not possible. All raw data will be stored on board. Less than 100 Gbytes of storage will be required for a 30-day ULDB balloon flight.

\section{SCIENTIFIC CAPABILITY}

We have generated simulations of the response and background for all three of the proposed payload configurations (Table 3) in order to determine the minimum detectable polarization (MDP) for several sources of interest (Crab, GRBs and solar flares).

\section{6..1 Background Estimation}

The primary background components in the $50-500 \mathrm{keV}$ energy band are expected to be the diffuse cosmic hard Xray background entering the instrument aperture and the leakage of atmospheric hard X-rays through the shields. We assume for now that prompt background due to cosmic-ray interactions in the instrument is effectively vetoed by the active shields and that activation is negligible during a relatively short balloon flight. We used a parameterization of the 
TABLE 3: Balloon Flight Schedule

\begin{tabular}{|c|c|c|c|c|}
\hline Flight & Date & Site & Configuration & Science Goal \\
\hline CONUS & Fall, 2011 & Ft. Sumner & Collimated $\left(10^{\circ}\right.$ FoV $)-4 \times 4$ array & Crab, Cyg X-1, Solar Flares \\
\hline LDB1 & Dec, 2012 & McMurdo & Un-collimated $\left(120^{\circ}\right.$ FoV $)-5 \times 5$ array & GRBs, Solar Flares \\
\hline LDB2 & Dec, 2013 & McMurdo & Un-collimated $\left(120^{\circ}\right.$ FoV $)-6 \times 6$ array & GRBs, Solar Flares \\
\hline
\end{tabular}

total cosmic plus atmospheric photon background [24] as the input for our simulation. This background is appropriate for a payload at an atmospheric depth of $3.5 \mathrm{~g} \mathrm{~cm}^{-2}$ at the approximate latitude of Palestine, TX or Ft. Sumner, NM. Additional simulations verify that, despite the high volume of plastic scintillator, GRAPE is relatively insensitive to background from elastic scattering of atmospheric neutrons on hydrogen, due to the requirement of a coincident trigger in the $\mathrm{CsI}(\mathrm{Na})$. We used the calculated atmospheric neutron spectrum [25], interpolated to an altitude of $3.5 \mathrm{~g} \mathrm{~cm}^{-2}$ and assumed for simplicity to be isotropic. We find a valid count rate from neutrons is less than $20 \%$ of the photon background. The background simulations (photons only, without neutrons) were validated using data from the EM1 balloon flight. The simulations incorporated effects of the shielding (both passive and active) as well as the aluminum pressure vessel. Although more detailed background modeling is currently in progress, the overall counting rate (50-300 keV) is comparable. This gives us confidence in our ability to provide a reasonable estimate of the background for other detector configurations.

To obtain background estimates for polar LDB flights, where we expect higher gamma-ray background levels due to lower magnetic rigidity, we compared data from $\mathrm{LaBr}_{3}$ scintillation detectors flown at Palestine (by UNH, as part of the GRAPE EM1 payload) and from McMurdo (by LSU, as a small piggyback device on the ATIC payload; Gary Case, private communications). A comparison of these data indicates that, for the energy range of 50-500 keV, the photon background is about a factor of 1.5-2.0 higher near the pole. Our sensitivity estimates given below for LDB flights therefore assume (conservatively) a background that is twice as high as that measured in our Palestine flight.

\subsection{CONUS Configuration}

For the first (CONUS) balloon flight, GRAPE will be configured as a 4x4 module array, with collimation of each module providing a $10^{\circ} \mathrm{FoV}$. The (on-axis) effective area and modulation factor as a function of energy for this configuration are shown in Figure 13. The average counting rate from the Crab was estimated using simulations based on an atmospheric depth of $3.5 \mathrm{~g} \mathrm{~cm}-2$ and a total observation time of 8 hours at an average Crab zenith angle of $30^{\circ}$ (typical numbers for the latitude of Ft. Sumner). From equation (4), and assuming an on/off observation strategy, we estimate a

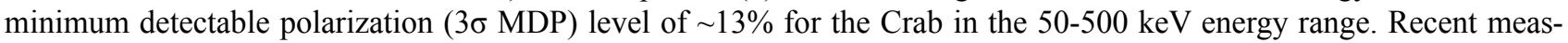
urements of the Crab nebula polarization with instruments on the INTEGRAL spacecraft indicate polarization levels in excess of $40 \%$ at GRAPE energies [26, 27]. These results indicate that GRAPE should be able to measure the polarization of the Crab nebula during a single source transit. (A second transit would push the MDP down to $\sim 9 \%$.) A somewhat better sensitivity will come from a phase-resolved analysis of the Crab flux that will allow us to separate the nebular component (which dominates the flux at these energies) from the pulsar. Observations of Cyg X-1, with an expected polarization level of less than a few percent, will provide a good null test for GRAPE. Observations of both sources will be possible for a 24-hour balloon flight, with two transits of the Crab for a 36-hour flight.

\subsection{LDB Configurations}

For the proposed LDB flights, GRAPE would be configured as an un-collimated array of polarimeter modules $(5 \times 5$ for the first LDB flight and $6 \times 6$ for the second LDB flight; Table 3). The simulated response parameters (modulation factor and effective area) for both configurations are shown in Figure 13 (on-axis) and Figure 14 (off-axis). A proper analysis of these data requires a crude knowledge of the source direction (to within $\sim 10-15^{\circ}$ ) due to the dependence of response parameters on incidence angle (Figure 14). Our baseline assumes that some source(s) of directional information for GRBs (e.g., GLAST) will be available. If necessary, a BATSE-like array of $\sim 4$ omnidirectional NaI(Tl) detectors will provide the necessary directional information. In the worst case, where no directional information is available, the weak dependence of modulation factor on incidence angle (c.f., Figure 14) will still allow us to obtain GRB polarization measurements. (By definition, solar flare directions are already known.) 
To estimate the sensitivity of the un-collimated configurations to GRB polarization we simulated the instrument response to a GRB viewed on-axis, but occurring at a zenith angle of $30^{\circ}$ (at an atmospheric depth of $3.5 \mathrm{~g} \mathrm{~cm}-2$ ). For input we used the spectrum of GRB021206, the very bright GRB for which a polarization level of $\sim 80 \%$ was reported [ 28 , 29]. The hard X-ray spectrum of this GRB was modified for the appropriate atmospheric absorption. Assuming a burst duration of 10 seconds, we calculated the $3 \sigma \mathrm{MDP}$ using Eq. 4. For a GRB of this magnitude, we expect a 50-500 keV $3 \sigma$ MDP value of $1.8 \%$ for a $5 \times 5$ array and $1.5 \%$ for a $6 \times 6$ array. Such a bright burst is only expected to be seen by a balloon instrument about once a year, however. Based on this result (for a GRB with 25-100 keV fluence of 4.8 x 10-4 erg cm-2), we used the 4th BATSE catalog to estimate the frequency and MDP for GRBs of various fluence levels as observed by the two LDB configurations of GRAPE (Table 4). The estimated frequencies were scaled for the relative FoV of BATSE and GRAPE. These estimates indicate that our observation program (two 30-day LDB flights) will result in the observation of $\sim 18$ GRBs with $3 \sigma$ MDP of $~ 50 \%$ or better. About 3 of these will be observed with an MDP of $\sim 15 \%$, which should allow us to study the energy-dependence of the GRB polarization within the GRAPE energy range. Collectively, these data will allow us : 1) to potentially confirm the high levels of polarization reported for some GRBs; 2) probe the jet geometry by distinguishing amongst alternative geometrical models; and 3) investigate the radiation mechanism(s) by studying the energy-dependence of the polarization.

For solar flares, we note that an effective area of $\sim 60 \mathrm{~cm}^{2}$ at $150 \mathrm{keV}$ (LDB2 configuration) is about $50 \times$ that of the RHESSI Be-scatter mode [30] and about 25x that of the SPR-N instrument on CORONAS-F [31, 32]. It is difficult to define a precise polarization sensitivity for a given class flare, since the flare classification is determined by the peak flux and the sensitivity depends on the integrated flux. For flights from McMurdo, exposure to the Sun is not optimum. The Sun's altitude during a typical LDB flight ranges between $10^{\circ}$ and $35^{\circ}$. For these flights, the GRAPE array would be pointed somewhat away from the zenith, in a direction towards the solar azimuth. To estimate the sensitivity to flare polarization in this "sun-following" mode, we simulated the instrument response to a solar flare occurring $30^{\circ}$ off-axis at a zenith angle of $65^{\circ}$, again assuming an atmospheric depth of $3.5 \mathrm{~g} \mathrm{~cm}^{-2}$. For input we used the peak hard X-ray spectrum

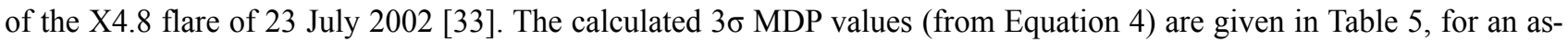
sumed flare duration of 120 secs. These data indicate that GRAPE, despite the non-optimum viewing conditions afforded by the geographic location of McMurdo, will be able to make useful polarization measurements of any M- or X-class

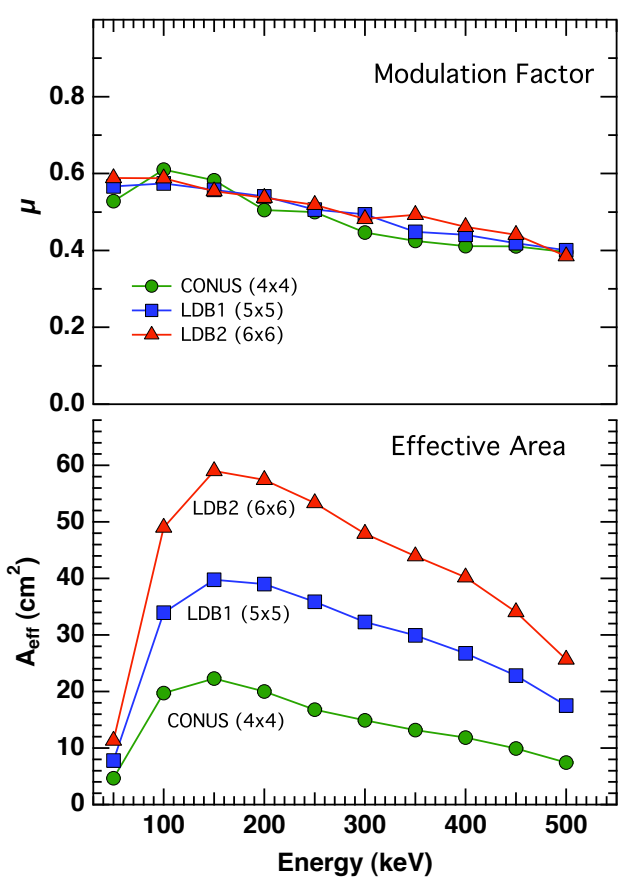

Figure 13: On-axis detection efficiency and modulation factor vs. energy for the three payload configurations.

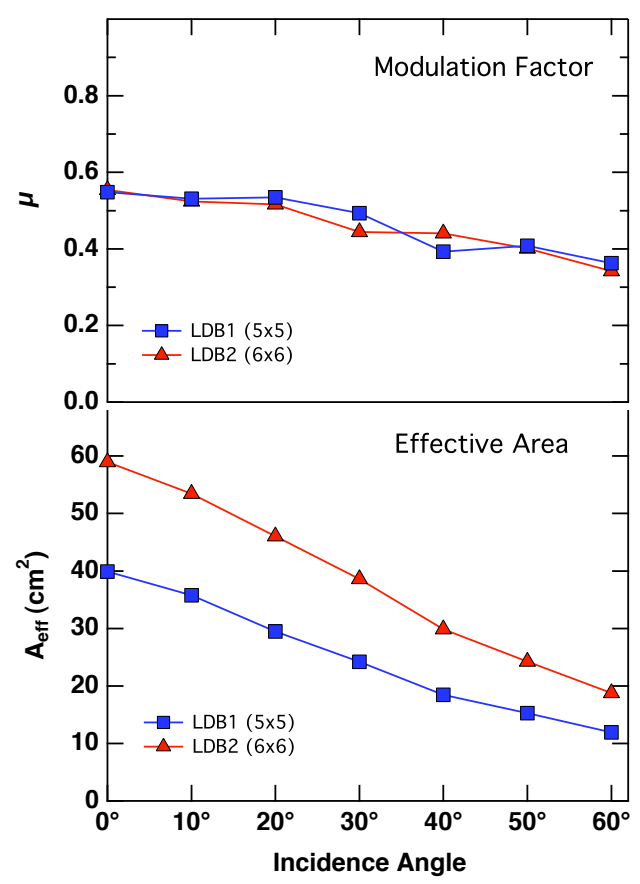

Figure 14: Off-axis detection efficiency and modulation factor (at $150 \mathrm{keV}$ ) for the LDB payload configurations. 
TABLE 4 - MDP for GRBs (50-500 keV)

\begin{tabular}{|c|c|c|c|}
\hline $\begin{array}{c}\text { Fluence } \\
(\mathbf{2 0 - 1 0 0} \mathrm{keV})\end{array}$ & GRB Freq & $\begin{array}{c}\text { LDB1 } \\
(\mathbf{5 \times 5})\end{array}$ & $\begin{array}{c}\text { LDB2 } \\
(\mathbf{6 x 6})\end{array}$ \\
\hline$>5 \times 10^{-5} \mathrm{erg} \mathrm{cm}^{-2}$ & $<0.1 /$ month & $5.4 \%$ & $4.5 \%$ \\
\hline$>1 \times 10^{-5} \mathrm{erg} \mathrm{cm}^{-2}$ & $0.5 /$ month & $12.1 \%$ & $10.1 \%$ \\
\hline$>5 \times 10^{-6} \mathrm{erg} \mathrm{cm}^{-2}$ & $1.5 /$ month & $17.2 \%$ & $14.3 \%$ \\
\hline$>1 \times 10^{-6} \mathrm{erg} \mathrm{cm}^{-2}$ & $6.0 /$ month & $38.4 \%$ & $32.0 \%$ \\
\hline$>5 \times 10^{-7} \mathrm{erg} \mathrm{cm}^{-2}$ & $9.0 /$ month & $54.3 \%$ & $45.0 \%$ \\
\hline
\end{tabular}

TABLE 5 - MDP for Solar Flares (50-500 keV)

\begin{tabular}{|l|c|c|c|c|}
\hline Configuration & $\mathbf{X} 4.8$ & $\mathbf{X 1}$ & $\mathbf{M} 5$ & $\mathbf{M 1}$ \\
\hline LDB1 & $2.3 \%$ & $5.0 \%$ & $7.3 \%$ & $15.9 \%$ \\
\hline LDB2 & $1.9 \%$ & $2.3 \%$ & $6.0 \%$ & $13.2 \%$ \\
\hline
\end{tabular}

solar flare. The peak rate of solar flares typically occurs 2-3 years after the peak sunspot number, with flare rates for $>\mathrm{M} 3$ class flares as high as 30 per month [34]. We might therefore expect as many as 40 flares to be observed by GRAPE with MDP larger than $20 \%$. These data, interpreted in the context of contemporaneous soft X-ray data (to define the magnetic field geometry), will provide a useful basis for studying the geometry of the electron acceleration process.

\section{SUMMARY}

Over the course of a series of LDB flights we would expect to achieve significant polarization measurements for several GRBs, and thus begin to distinguish the competing GRB models. To compile a large sample of measurements, however, the GRAPE instrument would need to fly on a space-based platform. One such platform, the POlarimeters for Energetic Transients (POET) mission, was proposed as a NASA Small Explorer mission in $2008[35,36]$. Such a mission would provide measurements with MDP of $20 \%$ or better for $\sim 80$ GRBs in two years and would definitively test GRB and solar flare emission models.

\section{BIBLIOGRAPHY}

1. Toma, K., et al., Statistical Properties of Gamma-Ray Burst Polarization. The Astrophysical Journal, 2009. 698(2): p. 1042-1053 (2009).

2. Bai, T. and R. Ramaty, Backscatter, anisotropy, and polarization of solar hard X-rays. Astrophysical Journal, 1978. 219: p. 705-726.

3. Leach, J. and V. Petrosian, The impulsive phase of solar flares. II - Characteristics of the hard X-rays. Astrophysical Journal, 1983. 269: p. 715-727.

4. Novick, R., Stellar and Solar X-Ray Polarimetry. Space Science Reviews, 1975. 18: p. 389.

5. Lei, F., A.J. Dean, and G.L. Hills, Compton Polarimetry in Gamma-Ray Astronomy. Space Science Reviews, 1997. 82: p. 309-388.

6. McConnell, M.L., et al., Development of a hard X-ray polarimeter for solar flares and gamma-ray bursts. Nuclear Science, IEEE Transactions on, 1998. 45(3): p. 910-914.

7. McConnell, M.L., et al. Development of a Hard X-Ray Polarimeter for Gamma-Ray Bursts. in American Institute of Physics Conference Series. 1998.

8. McConnell, M.L., et al., Development of a hard X-ray polarimeter for astrophysics. Nuclear Science, IEEE Transactions on, 1999. 46(4): p. 890-896.

9. McConnell, M. A Polarimeter for Studying Hard X-Rays from Solar Flares. in International Cosmic Ray Conference. 1999.

10. McConnell, M.L., et al., Recent laboratory tests of a hard x-ray solar flare polarimeter. Proceedings of the SPIE, 1999. 3764: p. 70-78.

11. McConnell, M.L., et al. A Modular Hard X-Ray Polarimeter for Solar Flares. in High Energy Solar Physics Workshop - Anticipating Hessi. 2000.

12. McConnell, M.L., et al. The Development of GRAPE, a Gamma Ray Polarimeter Experiment. in AIP Conf. Proc. 662: Gamma-Ray Burst and Afterglow Astronomy 2001: A Workshop Celebrating the First Year of the HETE Mission. 2003. 
13. McConnell, M.L., et al., Hard x-ray polarimeter for gamma-ray bursts and solar flares. Proceedings of the SPIE, 2003. 4851: p. 1382-1393.

14. McConnell, M.L., et al., Dedicated polarimeter design for hard x-ray and soft gamma-ray astronomy. Proceedings of the SPIE, 2004. 5165: p. 334-345.

15. Legere, J., et al., Developing a Compton polarimeter to measure polarization of hard x-rays in the 50-300 keV energy range. Proceedings of the SPIE, 2005. 5898: p. 413-422.

16. McConnell, M.L., et al. A hard X-ray polarimeter designed for transient astrophysical sources. 2005.

17. Bloser, P.F., et al., GRAPE - A Balloon-Borne Gamma-Ray Polarimeter Experiment. Chinese Journal of Astronomy and Astrophysics Supplement, 2006. 6: p. 393.

18. McConnell, M.L., et al. Prospects for GRB Polarimetry with GRAPE. in AIP Conf. Proc. 836: Gamma-Ray Bursts in the Swift Era. 2006.

19. Bloser, P.F., et al., Calibration of the Gamma-RAy Polarimeter Experiment (GRAPE) at a polarized hard X-ray beam. Nuclear Instruments and Methods in Physics Research Section A, 2009. 600(2): p. 424-433.

20. McConnell, M.L., et al., A Balloon-Borne Gamma-Ray Polarimeter for Gamma-Ray Bursts. GAMMA-RAY BURST: Sixth Huntsville Symposium. AIP Conference Proceedings, 2009. 1133: p. 61-63 (2009).

21. McMaster, W.H., Matrix Representation of Polarization. Reviews of Modern Physics, 1961. 33: p. 8-27.

22. Sturner, S.J., et al., MGEANT-A GEANT-Based Multi-Purpose Simulation Package for Gamma-Ray Astronomy Missions. The Fifth Compton Symposium, 2000. 510: p. 814.

23. Pendleton, G.N., et al. FiberGLAST: a scintillating fiber approach to the GLAST mission. in Proc. SPIE Vol. 3765 , p. 12-21, EUV, X-Ray, and Gamma-Ray Instrumentation for Astronomy X, Oswald H. Siegmund; Kathryn A. Flanagan; Eds. 1999.

24. Gehrels, N., Instrumental background in balloon-borne gamma-ray spectrometers and techniques for its reduction. Nuclear Instruments and Methods in Physics Research A, 1985. 239: p. 324-349.

25. Armstron.Tw, K.C. Chandler, and J. Barish, Calculations of Neutron-Flux Spectra Induced in Earths Atmosphere by Galactic Cosmic-Rays. Journal of Geophysical Research, 1973. 78(16): p. 2715-2726.

26. Dean, A.J., et al., Polarized Gamma-Ray Emission from the Crab. Science, 2008. 321(5893): p. 1183-1185.

27. Forot, M., et al., Polarization of the Crab Pulsar and Nebula as Observed by the INTEGRAL/IBIS Telescope. ApJ, 2008. 688: p. L29.

28. Boggs, S.E., et al., Testing Lorentz Invariance with GRB 021206. Astrophysical Journal, 2004. 611: p. L77-L80.

29. Coburn, W. and S.E. Boggs, Polarization of the prompt $\gamma$-ray emission from the $\gamma$-ray burst of 6 December 2002. Nature, 2003. 423: p. 415.

30. McConnell, M.L., et al., RHESSI as a Hard X-Ray Polarimeter. Solar Physics, 2002. 210: p. 125-142.

31. Bogomolov, A.V., et al., Polarization of Hard X-Rays in October-November, 2003 Solar Flares Observed Onboard CORONAS-F Satellite. Multi-Wavelength Investigations of Solar Activity, 2004. 223: p. 447.

32. Bogomolov, A.V., et al., Hard X-ray Radiation from Solar Flares in the Second Half of 2001: Preliminary Results of the SPR-N Experiment Onboard the Coronas-F Satellite. Solar System Research, 2003. 37: p. 112-120.

33. Holman, G.D., et al., Electron Bremsstrahlung Hard X-Ray Spectra, Electron Distributions, and Energetics in the 2002 July 23 Solar Flare. Astrophysical Journal, 2003. 595: p. L97-L101.

34. Temmer, M., A. Veronig, and A. Hanslmeier, Does solar flare activity lag behind sunspot activity? Solar Physics, 2003. 215(1): p. 111-126.

35. Hill, J.E., et al., POET: POlarimeters for Energetic Transients. 2008 NANJING GAMMA RAY BURST CONFERENCE. AIP Conference Proceedings, Volume 1065, pp. 331337 (2008). 2008.

36. McConnell, M.L., et al., GRB Polarimetry with POET. GAMMA RAY BURST: Sixth Huntsville Symposium. AIP Conference Proceedings, Volume 1133, pp. 6466 (2009). 2009. 